\title{
0 manejo dos resíduos florestais afeta a produção de biomassa e a sustentabilidade de povoamentos de Eucalyptus sp.
}

\section{Dione Richer Momolli e Mauro Valdir Schumacher}

${ }^{1}$ Universidade Federal de Santa Maria. Centro de Ciências Rurais. Programa de Pós-Gradução em Engenharia Florestal. Camobi. Santa Maria-RS, Brasil (CEP 97105-900). E-mail: dionemomolli@gmail.com.

${ }^{2}$ Universidade Federal de Santa Maria. Centro de Ciências Rurais. Departamento de Ciências Florestais. Campus da UFSM. Laboratório de Ecologia Florestal. Camobi. Santa Maria-RS, Brasil (CEP 97105-900).

Resumo. 0 resíduo da colheita florestal serve como fonte de matéria orgânica e nutrientes ao desenvolvimento das futuras rotações, além disso, atua na proteção do solo evitando a erosão, a exposição direta aos raios solares e a compactação, aumentando a capacidade de retenção hídrica. A biota do solo possui o papel de decompor e mineralizar os nutrientes presentes na matéria orgânica. Através desse processo acelera-se a ciclagem dos nutrientes, liberando-os para posterior absorção pelas raízes das árvores. 0 descascamento da tora a campo é uma forma de manejo que minimiza o percentual de nutrientes exportados. 0 objetivo dessa revisão bibliográfica é abordar os efeitos do manejo dos resíduos da colheita na produção de biomassa e na sustentabilidade das plantações de eucalipto.

Palavras-chave: Nutrição florestal; Sustentabilidade; Silvicultura.

Abstract. The harvest residue management affects biomass production and sustainability of Eucalyptus sp. stands. The forest harvesting residue serves as a source of organic matter and nutrients to the development of future rotations, besides, it acts in the protection of the soil avoiding the erosion, the direct exposure to the solar rays and the compaction, increasing the capacity of water retention. Soil biota has the role of decomposing and mineralizing the nutrients present in organic matter. This process accelerates the nutrient cycling, releasing them for later absorption by the roots of the trees. Debarking of the stem in field is a form of management that minimizes the percentage of nutrients exported. The objective of this bibliographic review is to address the effects of harvest residue management on biomass production and the sustainability of Eucalyptus stands.

Keywords: Forest nutrition; Sustainability; Forestry.
Recebido: $15 / 01 / 2019$

Aceito:

22/04/2019

Publicado:

30/04/2019

Acesso aberto

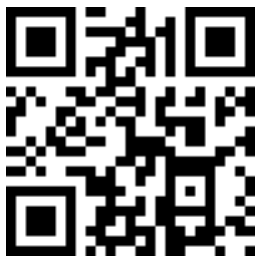

ORCID

(D) 0000-0001-7798-6355 Dione Richer Momolli

(D) 0000-0001-6926-6200

Mauro Valdir

Schumacher 


\section{Introdução}

Tendo em vista a maximização dos produtos oriundos da floresta, aliada a busca por fontes energéticas mais sustentáveis, empresas do setor florestal avistam como oportunidade a reutilização dos chamados "resíduos da colheita" formado por folhas, ramos e casca, á produção de pellets, servindo assim como subproduto de base energética ás indústrias para 0 beneficiamento da madeira em serrarias, na obtenção de papel e celulose ou usado na obtenção de eletricidade (Gustavsson et al., 2015).

Estudos mostram projeções de aumento significativo do uso de fontes de energia mais sustentáveis frente aos combustíveis fósseis. A bioenergia oriunda dos resíduos florestais (folhas, galhos, casca e etc.), tem sido o carro chefe dessa nova tendência mundial (IEA, 2011; Farine et al., 2012).

Alguns aspectos econômicos corroboraram para o aumento da demanda de combustíveis oriundos dos resíduos florestais. Inicialmente destacamos a crise do óleo que gerou aumento nos preços desse produto nos anos compreendidos entre 1970 e 1980, diante dessa situação, curtas rotações foram adotadas (Ranger e Nys, 1986; Díaz-Yáñez et al., 2013), além da remoção dos resíduos da colheita para geração de energia (Nunez-Regueira et al., 2005; Díaz-Yáñez et al., 2013).

A remoção dos resíduos da colheita, facilita ainda as operações de implantação de nova rotação e reduzem o risco eminente de incêndios. Contudo, esse material possui elevados teores de nutrientes e matéria orgânica (Sankaran et al., 2005; Nambiar, 2010), e a sua remoção repetida acarreta em déficits nutricionais e de matéria orgânica ao solo, gerando diminuição da atividade biológica, redução da umidade e compactação do mesmo (Kumaraswamy et al., 2014; Achat et al., 2015).

Estudos conduzidos por

Mendham et al. (2003) e Mendham et al. (2014) relatam uma diminuição do crescimento e da produtividade em segunda e terceira rotação no sudeste da Austrália. Além disso, a transformação dos resíduos florestais em bioenergia, libera o carbono que antes fora estocado no processo de formação da energia, (Zeng et al., 2012), por isso, deve-se minimizar os impactos da remoção exportando apenas do componente madeira. Assim, o entendimento da dinâmica nutricional em ecossistemas florestais (Schumacher e Viera, 2015), subsidia informações para um manejo correto dos resíduos visando a mitigar os impactos oriundos da colheita na fertilidade do solo (Achat et al., 2015).

No Brasil, os plantios de eucalipto estão concentrados em áreas de baixo potencial para agricultura e em solos caracterizados por uma baixa fertilidade natural (Gonçalves et al., 2013), por isso, objetivou-se na presente revisão bibliográfica avaliar os efeitos da remoção dos resíduos na produção de biomassa e crescimento de Eucalyptus sp.

0 sucesso da plantação de espécies consideradas de rápido crescimento é dependente do estoque de nutrientes no solo (Laclau et al., 2005). Quando esses nutrientes são ofertados em baixas quantidades, como é o caso dos solos arenosos, as plantações tornam-se dependentes da aplicação de fertilizantes (Bouillet et al., 2004; Mendham et al., 2009).

Nas áreas de reforma, onde há implantação de nova rotação, além da análise química do solo faz se necessário conhecer a quantidade de nutrientes que foi exportada pela colheita anterior. Somente dessa forma é possível garantir a sustentabilidade da produção ao longo das rotações. Sob o ponto de vista do estoque de nutrientes existente no solo, Gonçalves et al. (1996) recomendam a adubação nitrogenada com base nas classes de matéria orgânica do solo e P e $\mathrm{K}$ baseado no percentual de argila e $\mathrm{P}$ e $\mathrm{K}$ no mesmo.

Os solos arenosos possuem baixa retenção de nutrientes e alta drenagem, tornando-se mais susceptível á lixiviação dos nutrientes (Mitchell e Smethurst, 2008). Diante disso, o manejo da fertilização, doses, quantidades e épocas 
de aplicação, torna-se ferramentas essenciais para que se evitem perdas e os resultados sejam maximizados.

Em estudos de manejo de fertilização em plantações de eucaliptos em solos arenosos, Silva et al. (2013) concluíram que o gênero responde positivamente ás doses de fertilizantes. Para os autores a aplicação parcelada de $\mathrm{N}$ e K após 2 anos do plantio (prática comum no Brasil), não aumentou a produção quando comparada com uma única aplicação $\left(80 \mathrm{~kg} \mathrm{ha}^{-1} \mathrm{~N}\right.$; $32 \mathrm{~kg} \mathrm{ha}^{-1}$ P; $\left.106 \mathrm{~kg} \mathrm{ha}^{-1} \mathrm{~K}\right) 3$ meses após o plantio. Os autores sugerem que as empresas reduzam o número de aplicações em solos sob essas condições.

Menegale et al. (2016) estudaram o efeito de diferentes intensidades de fertilização no estoque de nutrientes no solo e seus resultados mostraram que $\mathrm{N} \mathrm{e}$ $P$ possuem uma função importante para nutrição das florestas, sendo que a redução dos mesmos especialmente de (P), pode afetar o sistema de produção sustentável, sendo que para os autores, a ausência do mesmo na fertilização, aliado a retirado dos resíduos mais a camada de serapilheira, resultou em uma biomassa $25 \%$ inferior quando comparado ao tratamento em que houve manutenção dos resíduos mais fertilização padrão.

\section{biomassa}

Crescimento e produção de

Sabe-se que a manutenção do resíduo orgânico sobre o solo é uma prática que além de minimizar a exportação de nutrientes, mantém a fertilidade do solo (Zerpa et al., 2010; Mendham et al., 2014). Contudo há de se considerar ainda a compactação gerada ao solo causada pelo trafego de maquinas agrícolas (Seixas, 2002).

A compactação do solo dificulta o crescimento do sistema radicular pela redução dos macroporos, capacidade de retenção hídrica e disponibilidade de oxigênio no mesmo (Seixas, 2002). Após verificado compactação do solo e comprometimento do desenvolvimento do sistema radicular, há diminuição significativa da produtividade de madeira, como constatado por Dedecek e
Gava, (1997) em povoamento de Eucalyptus grandis aos sete anos de idade. Os pesquisadores avaliaram a produtividade em três faixas, manutenção da galhada sem tráfego (a), galhada com empilhamento de madeira sem tráfego (b) e tráfego (c). Houve uma redução de 58\% em Latossolo vermelhoamarelo distrófico e 33\% em Latossolo vermelho distrófico no volume de madeira $\mathrm{m}^{3}$ ha $^{-1}$ do tratamento (a) para o (b) respectivamente.

Epron et al. (2015) em um povoamento de eucaliptos em solos arenosos, constataram que o tratamento em que teve remoção do resíduo orgânico, houve uma redução na média de altura, área basal e carbono em segunda e terceira rotação, frente os tratamentos de colheita da madeira com casca e sem casca. Os valores de altura média quando se removeu o resíduo orgânico foram de 26,1; 20,9; e 19,1 m e de $12,9,9,9$ e 7,8 $\mathrm{m}^{2}$ ha-1 $^{-1}$ de área basal ao final da $1^{\underline{a}}, 2^{\underline{a}}$ e $3^{\underline{a}}$ rotação para 0 tratamento em que houve remoção da madeira com casca e resíduos.

\section{Biomassa e nutrientes}

A variação do estoque de nutrientes nos componentes da biomassa é condicionado principalmente pela maturidade do povoamento. Witschoreck e Schumacher (2015) mostram que as folhas contribuem com cerca de $30 \%$ dos macronutrientes alocados aos dois anos de idade, esse percentual cai para $22 \%$, $17 \%, 15 \%, 10 \%$ e $9 \%$ aos $3,4,5,6$ e 7 anos de idade. Em contrapartida, o volume em madeira aumenta gerando maiores estoques de nutrientes, $15 \%$, $23 \%, 27 \%, 27,3 \%, 37 \%$ e $39 \%$ aos $2,3,4$, 5,6 e 7 anos de idade (Tabela 1). Os autores em seus estudos mostram ainda que quando exportada apenas a madeira, apenas $47,8 \%$ dos nutrientes são removidos do sítio, restando ainda cerca de $52,2 \%$ dos nutrientes.

Hérnandes et al. (2009) em plantio de Eucalyptus dunnii com nove anos de idade localizado no Uruguai mostrou que $61 \%$ da quantidade de biomassa foi exportada pela colheita, no entanto, apenas 20,8\% dos nutrientes 
foram removidos do sítio. Esse resultado pode ser atribuído a menor concentração de nutrientes na madeira $0,9 \mathrm{~g} \mathrm{~kg}^{-1}$, valores esses bastante inferiores se comparado ao teor de nitrogênio na folha 14,2 $\mathrm{g} \mathrm{kg}^{-1}$. Os autores ainda em seus estudos observaram que a casca contribui com mais da metade dos nutrientes deixados pela colheita, servindo dessa forma como importante fonte nutricional às rotações futuras.

Tabela 1. Estoque de nutrientes nos componentes da biomassa de Eucalyptus em diferentes idades.

\begin{tabular}{|c|c|c|c|c|c|c|c|c|}
\hline Idade & Comp. & $\begin{array}{l}\text { Biomassa } \\
\left(\mathrm{Mg} \mathrm{ha}^{-1}\right)\end{array}$ & N & $\mathbf{P}$ & $\begin{array}{c}\mathrm{K} \\
\mathrm{kg} \mathrm{ha} \mathbf{A}^{-1}\end{array}$ & Ca & Mg. & Autor \\
\hline \multirow{6}{*}{4,0} & $\mathrm{~F}$ & 5,3 & 93,5 & 5,4 & 32,0 & 36,3 & 12,9 & \multirow{5}{*}{ Guimarães et al. (2015) } \\
\hline & G & 13,4 & 37,2 & 2,9 & 56,2 & 83,5 & 20,5 & \\
\hline & $\mathrm{C}$ & 9,1 & 32,7 & 5,2 & 35,8 & 179,9 & 43,8 & \\
\hline & M & 76,7 & 73,1 & 6,1 & 98,8 & 36,4 & 49,6 & \\
\hline & $\mathrm{R}$ & 17,4 & 50,0 & 5,7 & 30,4 & 113,1 & 19,7 & \\
\hline & Total & 121,9 & 286,5 & 25,3 & 253,1 & 449,0 & 146,5 & \multirow{6}{*}{ Carvalho et al. (2015) } \\
\hline \multirow{5}{*}{4,5} & $\mathrm{~F}$ & 4,1 & 71,3 & 4,4 & 27,7 & 32,1 & 7,4 & \\
\hline & G & 7,7 & 35,9 & 3,7 & 43,6 & 67,7 & 13,2 & \\
\hline & $\mathrm{C}$ & 5,7 & 24,4 & 2,9 & 38,7 & 89,1 & 13,3 & \\
\hline & M & 45,8 & 73,0 & 6,1 & 112,9 & 63,5 & 15,3 & \\
\hline & $\mathrm{R}$ & 11,6 & 46,5 & 3,1 & 42,4 & 101,4 & 14,8 & \\
\hline & Total & 74,8 & 251,2 & 20,2 & 265,2 & 353,8 & 63,9 & \multirow{5}{*}{ Dick et al. (2016) } \\
\hline \multirow{4}{*}{5,0} & $\mathrm{~F}$ & 3,6 & 61,7 & 3,9 & 22,3 & 35,0 & 10,3 & \\
\hline & G & 6,5 & 25,3 & 2,3 & 28,6 & 63,1 & 11,5 & \\
\hline & $\mathrm{C}$ & 7,9 & 28,5 & 4,3 & 41,0 & 128,6 & 22,9 & \\
\hline & M & 40,8 & 60,0 & 7,8 & 79,2 & 51,4 & 24,5 & \\
\hline & Total & 58,8 & 175,5 & 18,2 & 171,0 & 278,1 & 69,1 & \multirow{19}{*}{$\begin{array}{l}\text { Witschoreck and } \\
\text { Schumacher (2015); } \\
\text { Witschoreck (2014) }\end{array}$} \\
\hline \multirow{6}{*}{5,0} & $F$ & 5,7 & 124,2 & 11,6 & 35,8 & 47,8 & 15,0 & \\
\hline & G & 8,4 & 33,1 & 6,2 & 30,7 & 74,3 & 14,7 & \\
\hline & $\mathrm{C}$ & 10,4 & 38,9 & 6,7 & 36,7 & 195,8 & 34,6 & \\
\hline & M & 115,3 & 170,5 & 23,1 & 139,3 & 72,0 & 31,5 & \\
\hline & $\mathrm{R}$ & 40,4 & 127,3 & 10,1 & 80,5 & 202,3 & 34,3 & \\
\hline & Total & 180,2 & 494,1 & 57,7 & 323,0 & 592,1 & 130,0 & \\
\hline \multirow{6}{*}{6,0} & $\mathrm{~F}$ & 4,8 & 101,3 & 6,8 & 32,9 & 30,1 & 13,5 & \\
\hline & G & 7,6 & 30,9 & 4,1 & 29,7 & 63,9 & 15,0 & \\
\hline & $\mathrm{C}$ & 13,2 & 53,1 & 7,1 & 49,8 & 195,3 & 51,4 & \\
\hline & M & 154,0 & 265,7 & 27,4 & 194,6 & 169,5 & 46,0 & \\
\hline & $\mathrm{R}$ & 43,1 & 130,6 & 10,5 & 80,9 & 221,2 & 45,6 & \\
\hline & Total & 222,6 & 581,5 & 55,8 & 387,8 & 680,0 & 171,5 & \\
\hline \multirow{6}{*}{7,0} & $F$ & 4,7 & 76,9 & 5,0 & 30,9 & 30,8 & 12,1 & \\
\hline & G & 8,9 & 34,5 & 3,5 & 38,0 & 66,1 & 17,0 & \\
\hline & $\mathrm{C}$ & 15,3 & 54,5 & 7,4 & 55,0 & 241,0 & 53,7 & \\
\hline & M & 178,7 & 252,6 & 32,2 & 209,9 & 163,3 & 57,7 & \\
\hline & $\mathrm{R}$ & 32,5 & 110,3 & 9,0 & 60,5 & 170,9 & 27,2 & \\
\hline & Total & 240,0 & 528,9 & 57,0 & 394,2 & 672,1 & 167,7 & \\
\hline
\end{tabular}

Schumacher e Caldeira (2001) mostram que as toras quando descascadas a campo, cerca de $60 \%$ dos macronutrientes são exportados. Em contrapartida, quando não realizado o descascamento, há um incremento substancial de nutrientes removidos via colheita florestal. 
$\mathrm{Na}$ Figura 1 observamos os percentuais de biomassa e de nutrientes nos diferentes componentes e idades. Os dados são provenientes da Tabela 1. Notamos que do $4^{\circ}$ ao $7^{\circ}$ ano de idade os percentuais de madeira oscilaram de $63 \%$ a $74 \%$, já o total de nutrientes para o mesmo componente variou de $23 \%$ a $39 \%$. Esses resultados reforçam que embora a madeira represente mais da metade da biomassa exportada, no máximo $39 \%$ dos nutrientes serão removidos.

\begin{abstract}
Considerando que $\quad 0$ descascamento não seja realizado no sítio, o percentual de biomassa exportada passa dos $74 \%$ para $80 \%$. 0 maior impacto, entretanto, está no percentual de exporte de nutrientes que passa dos $39 \%$ para $62 \%$ no sétimo ano. Sob ponto de vista da sustentabilidade dos povoamentos florestais, é desejável que apenas o componente madeira seja colhida, minimizando dessa forma o déficit nutricional do sítio.
\end{abstract}

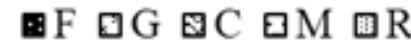
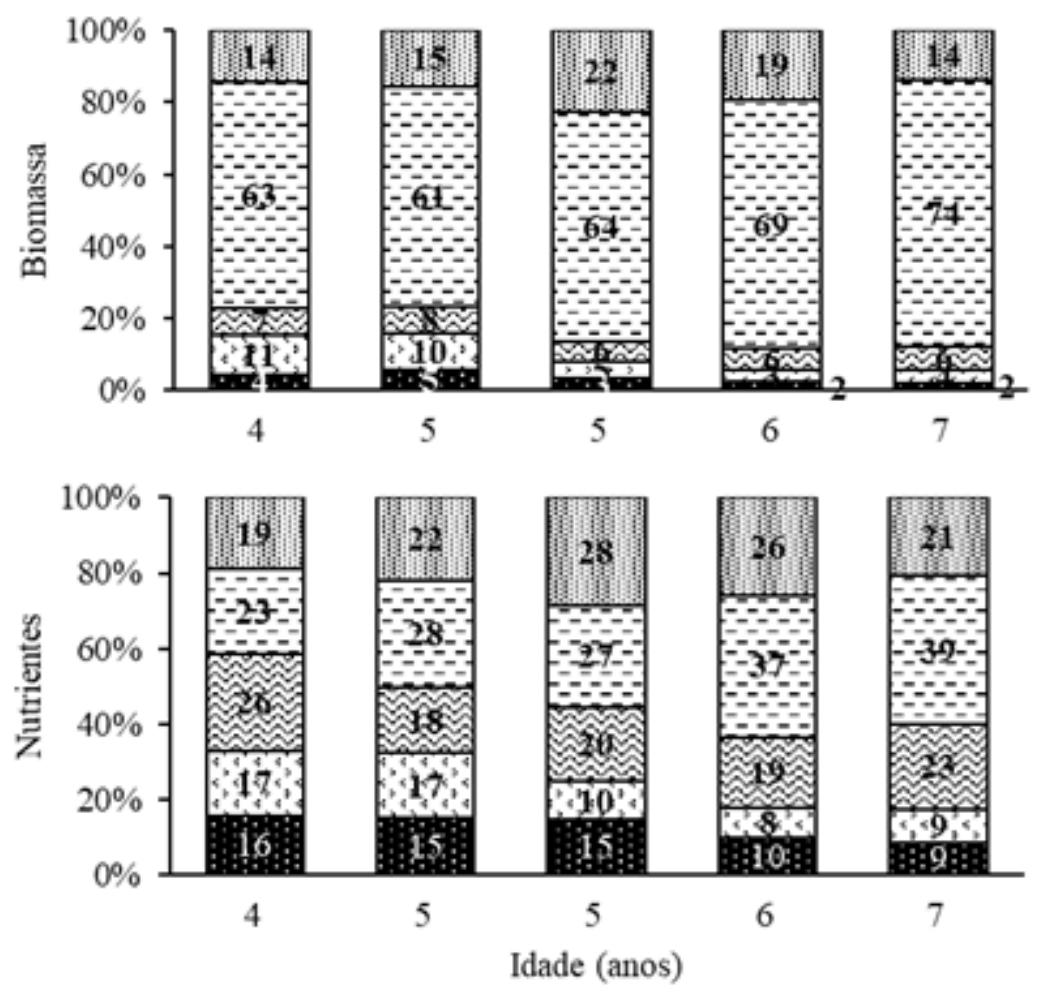

Figura 1. Percentual de biomassa e nutrientes em diferentes frações e idades de povoamentos de Eucalyptus sp.

\section{Decomposição dos resíduos}

A taxa de decomposição de tecidos vegetais é condicionada pelas variáveis climáticas, além da interação entre decompositores e qualidade do substrato (Brady e Weil, 2013; Purahong et al., 2014). A colheita florestal gera diferentes resíduos, entre eles, folhas, galhos grossos e finos e casca.

Ferreira et al. (2016) avaliaram a taxa de decomposição dos resíduos da colheita em Eucalyptus sp. em diferentes sítios no Brasil e concluíram que a liberação dos nutrientes é mais 
influenciada pelo manejo e pelas características físicas e químicas dos resíduos do que pelas características climáticas e do solo. 0 estudo conduzido pelos autores mostrou ainda que a ausência da casca na superfície do solo aumenta a imobilização de nutrientes. Com isso, a manutenção dos resíduos, principalmente em solos ácidos e pobres em nutrientes, reduz a necessidade de retenção de grandes quantidades de nutrientes por parte dos microrganismos.

A casca acumula grandes quantidades de cálcio em sua estrutura e esse elemento é pouco retranslocado dentro da planta (Vergutz et al., 2012), sendo assim, o descascamento a campo reduz a pressão sobre reservas de $\mathrm{Ca}$ e Mg no solo (Santana et al., 2008).

Rocha et al. (2016) avaliaram a taxa de decomposição dos resíduos da colheita de Eucalyptus grandis submetido a diferentes tratamentos e constataram que a taxa de decomposição inicial foi mais acelerada, devido principalmente pela degradação de carboidratos não estruturais e baixo peso molecular de fenóis (Hättenschwiler e Jorgensen, 2010; Hättenschwiler et al., 2011). Rocha et al. (2016) dividem ainda a decomposição em dois estágios: o primeiro ao qual as folhas e galhos finos são nutricionalmente ricos em componentes, que por sua vez, aceleram a taxa de decomposição e o segundo estágio em que restam apenas galhos grossos e casca, esses, com teores mais elevados de lignina retardando a degradação.

\section{Solo: densidade, matéria orgânica, nutrientes}

De acordo com Lal (2009) e Achat (2015), há inúmeras vantagens as quais a manutenção dos resíduos da colheita podem gerar incremento ou manutenção dos teores de matéria orgânica no solo, redução da densidade aparente, aumento da infiltração da água da chuva, melhora considerável contra erosão, redução de extremos térmicos e aumento da biomassa microbiana e consequente mineralização dos nutrientes.
Uma possível forma de avaliar o impacto dos resíduos da colheita sobre o solo, dá-se por meio da análise química da solução do solo. Essa técnica consiste na instalação sondas para sucção da solução do solo. Em estudo desenvolvido por Ring et al. (2016), sondas foram alocadas a $50 \mathrm{~cm}$ de profundidade, em solo submetido dois manejos diferentes de colheita: convencional e árvore inteira. Os resultados obtidos pelos autores, mostraram que as concentrações dos elementos avaliados tenderam a uma diminuição sob regime de colheita de árvore inteira. $\mathrm{NO}_{3}{ }^{-}-\mathrm{N}$, foi significativamente inferior sob regime de colheita de árvore inteira. Outros estudos corroboram com os resultados obtidos, como é o caso de Thiffault et al. (2011).

Os resultados encontrados por Ring et al. (2016) mostraram um aumento nos teores de $\mathrm{N}$ até a $2^{\mathrm{a}}$ estação do ano, seguido de uma redução á níveis próximos a zero já na 5 a estação após corte raso. Para Hedwall et al. (2014), o crescimento da vegetação após o corte raso, tende a reduzir as concentrações de $\mathrm{N}$ na solução do solo, justificando tais resultados.

$$
\text { Outros estudos foram }
$$

desenvolvidos como é o caso de Mendes et al. (2018) avaliando a compactação do solo após a colheita florestal de um povoamento de Eucalyptus saligna Smith aos 40 anos de idade. Os autores observaram que há diferença estatística entre o modo convencional mecanizado e o sistema de colheita com impacto reduzido. A remoção dos resíduos da colheita aliada a intensa mecanização, causa maior compactação do solo quando comparado ao sistema de colheita de impacto reduzido. Essa compactação por sua vez, desequilibra o ambiente, permitindo em alguns casos a dominância de arbustos ou vegetação mais rasteira frente a espécies arbóreas.

Diversos estudos têm mostrado redução da compactação do solo pela presença de camadas de resíduos florestais (Wronski, 1990; Seixas et al., 1995). Durante o transporte de madeira de Eucalyptus grandis com trator + grua + carreta, Seixas et al. (1998) observaram 
uma redução de $56 \%$ na compactação do solo ao manter $10 \mathrm{~kg} \mathrm{~m}^{2}$ de resíduos (casca, galhos e folhas).

Com relação a matéria orgânica, Rocha et al. (2016) constataram uma redução dos teores até o final da rotação, aos oito anos de idade em povoamento de Eucalyptus grandis, sob manejo dos resíduos e aplicações de fertilizantes. Outros estudos conduzidos em regiões temperadas encontraram redução do teor de matéria orgânica apenas nos anos iniciais.

Outra variável que é afetada de acordo com regime de colheita adotado é a umidade relativa do solo. Quatro diferentes sistemas de tratamento dos resíduos foram avaliados por Gonçalves et al. (1999) em povoamento de Eucalyptus grandis. Os autores concluíram que que até os $30 \mathrm{~cm}$ de profundidade registrou-se uma redução percentual de umidade relativa para os tratamentos com remoção total dos resíduos e os resíduos queimados. Com relação à temperatura a $2,5 \mathrm{~cm}$ de profundidade no solo, nitidamente observa-se maior amplitude térmica quando os resíduos foram totalmente removidos. A manutenção dos resíduos protege o solo da exposição direta a raios solares e mantem a umidade e a temperatura mais constante.

O clima com temperaturas mais amenas, torna a atividade biológica mais lenta, que por sua vez auxilia na estabilização da MO do solo (Mendham et al., 2002; Laclau et al., 2010). Os teores de areia e argila influenciam o comportamento da MO no solo. Em solos arenosos devido a menor formação de complexos organominerais, a matéria orgânica torna-se menos estável. Em estudo desenvolvido por Mendham et al. (2003) com Eucalyptus globulus na Austrália, os autores encontraram que a remoção dos resíduos da colheita reduziu o teor de M. 0 . no solo até os sete anos, em contrapartida, o mesmo não foi observado quando o povoamento foi estabelecido em solo com maiores teores de argila.

\section{Atividade biológica do solo}

O solo pode ser considerado como um sistema vivo, o qual é responsável por um dos principais reservatórios biológicos (Cardoso e Andreote, 2016). 0 mesmo está estruturado de forma heterogênea. Esta variação está condicionada principalmente ao acúmulo de material orgânico com elevadas fontes nutricionais o qual cria micro-habitats propícios ao desenvolvimento da fauna do solo (Moreira e Siqueira, 2002; Duchicela et al., 2013; Vos et al., 2013).

Kumaraswamy et al. (2014) avaliaram o carbono da biomassa microbial em dois sítios, planície com a espécie de Eucalyptus tereticornis e terras altas com a espécie Eucalyptus grandis. 0 estudo conduzido pelos autores, mostrou que em terras altas, o teor de carbono na biomassa microbial foi superior quando comparado às planícies. Os autores ressaltam que os resultados se devem a taxa de decomposição mais acentuada nas planícies, bem como, as altas temperaturas e precipitações, que promoveram a rápida taxa de decomposição.

Após a colheita e manutenção dos resíduos sobre o solo, observa-se elevadas taxas de decomposição. Essa taxa se deve principalmente está associada ao aumento da atividade microbiana e como consequência, imobilização de parte dos nutrientes por essa biota (Rocha et al., 2016).

De acordo com Gonçalves et al. (2002), a população de microrganismos tende a aumentar por ocasião da presença de compostos ricos em energia recém acumulados no solo pelo resíduo florestal. Entre eles destacamos as bactérias e os fungos, decompositores de fontes imediatamente assimiláveis e de compostos ricos em lignina respectivamente. A micro, meso e macrofauna do solo também possui papel importante. Elas são responsáveis principalmente pela fragmentação do material, isso aumenta a área específica e auxilia a posterior ação dos microrganismos anteriormente mencionados. 


\section{Considerações finais}

O impacto da colheita e exporte de nutrientes vai variar com a idade do povoamento e o regime de colheita empregado. 0 processamento do fuste a campo permite com que uma maior quantidade de resíduos e nutrientes permaneçam no sítio, contudo há de prestar atenção na disposição do mesmo, evitando o enleiramento.

Embora a aplicação de fertilizantes inorgânicos seja indispensável, nem sempre são capazes de suprir as necessidades das rotações futuras. Aspectos da biologia do solo e matéria orgânica acabam sendo prejudicados, visto que a microbiologia do solo é responsável pela transformação da matéria orgânica através da decomposição e a fertilização compensa apenas o deficit nutricional.

De forma geral os estudos mostram que a remoção dos resíduos da colheita implica em déficits nutricionais às rotações subsequentes. A camada de resíduos evita a excessiva compactação do solo pelo tráfego das maquinas e protege contra exposição direta dos raios solares, ambos auxiliam na manutenção da umidade do solo, contenção de erosão e lixiviação dos nutrientes. A atividade biológica do solo é estimulada a medida em que há tecido vegetal. A mesma encontra um microambiente adequado ao desenvolvimento, acelerando a mineralização.

\section{Conflito de interesses}

Os autores declaram não haver conflito de interesses.

\section{Referências}

Achat, D. L.; Deleuze, C.; Landmann, G.; Pousse, N.; Ranjer, J.; Augusto, L. Quantifying consequenses of removing harvesting residues on forest soils and tree growth: A meta-analysis. Forest Ecology and Management, v. 348, p. 124-141, 2015. https://doi.org/10.1016/j.foreco.2015.03.042
Bouillet, J. P.; Safou-Matondo, R.; Laclau, J. P.; Nzila, J. D.; Ranger, J.; Deleporte, P. Pour une production durable des plantations d'eucalyptus au Congo: la fertilisation. Bois et Forêts des Tropiques, v. 279, n. 1, p. 2335, 2004.

Brady, N. C.; Weil, R. R. Elementos da natureza e propriedades dos solos. Porto Alegre: Bookman, 2013.

Cardoso, E. J. B. N.; Andreote, F. D. Microbiologia do solo. 2. ed. Piracicaba: Esalq, 2016.

Dedecek, R. A.; Gava, J. L. Compactação do solo pela colheita de eucalipto: sua avaliação e efeito na produtividade da rebrota. Anais do IUFRO Conference on Silviculture and Improvement of Eucalyptus, Salvador, v. 3, p. 63-68, 1997.

Díaz-Yáñez, O.; Mola-Yudego, B.; Anttila, P.; Röser, D.; Asikainen, A. Forest chips for energy in Europe: Current procurement methods and potentials. Renewable and Sustainable Energy Reviews, v. 21, p. 562-571, 2013. https://doi.org/10.1016/ j.rser.2012.12.016

Dick, G.; Schumacher. M. V.; Momolli, D. R.; Santos, J. C.; Ludvichak, A. P.; Guimarães, C. G; Souza, H. P. Quantificação da biomassa e nutrientes em um povoamento de Eucalyptus dunnii Maiden estabelecido no Bioma Pampa. Ecologia e Nutrição Florestal, v. 4, n. 1, p. 1-9, 2016.

Duchicela, J.; Sulivan, T. S; Bontti, E.; Bever, J. D. Soil aggregate stability increase is strongly related to fungal community succession along an abandoned agricultural field chronosequence in the Bolivian Altiplano. Journal of Applied Microbiology, v. 50, p. 1266-1273, 2013.

Epron, D.; Mouanda, C.; Mareschal, L.; Koutika, L. S. Impact of organic residue management on the soil $\mathrm{C}$ dynamics in $\mathrm{a}$ tropical eucalypt plantation on a nutrientepoor: Sandy soil after three rotations. Soil Biology and Biochemistry, v. 85, p.183-189, 2015.

Farine, D.; O'Connell, D.; Raison, R.; May, B.; O'Connor, M.; Crawford, D.; Herr, A.; Taylor, J.; Jovanovic, T.; Campbell, P.; Dunlop, M.; Rodriguez, L.; Poole, M.; Braid, A.; Kriticos, D. An assessment of biomass for bioelectricity and biofuel, and for greenhouse gas emission 
reduction in Australia. Global Change Biology Bioenergy, v. 4, p. 148-175, 2012. https://doi.org/10.1111/j.1757-1707. 2011.01115.x

Ferreira, G. W. D.; Soares, E. M. B.; Oliveira, F. C. C.; Silva, I. R.; Dungait, J. A. J.; Souza, I. F.; Vergütz, L. Nutrient release from decomposing Eucalyptus harvest residues following simulated management practices in multiple sites in Brazil. Forest Ecology and Management, v. 370, p. 1-11, 2016.

Gonçalves, J. L. M.; Alvares, C. A.; Higa, A. R.; Silva, L. D.; Alfenas, A. C.; Stahl, J.; Ferraz, S. F. D.; Lima, W. D. P.; Brancalion, P. H. S.; Hubner, A.; Bouillet, J. P. D.; Laclau, J. P.; Nouvellon, Y.; Epron, D. Integrating genetic and silvicultural strategies to minimize abiotic and biotic constraints in Brazilian eucalypt plantations. Forest Ecology and Management, v. 301, p. 6-27, 2013.

Gonçalves, J. L. M.; Poggiani, F.; Stape, J. L.; Serrano, M. I. P. S.; Mello, S. L. M.; Mendes, K. C. F. S.; Gava, J. L.; Benedetti, V. Eucalypt plantations in the humid tropic: São Paulo, Brazil. In: Nambiar, E. K. S.; Cossalter, C.; Tiarks, A. (Eds.). Site management and productivity in tropical plantation forests. Pietermaritzburg, South Africa: CIFOR, 1999. p. 5-12.

Gonçalves, J. L. M.; Stape, J. L.; Wichert, M. C. P.; Gava, J. L. Manejo dos resíduos vegetais e preparo do solo. In: Gonçalves, J. L. M.; Stape, J. L. (Eds.). Conservação e cultivo de solos para plantações florestais. Piracicaba: IPEF, 2002. p. 131-204.

Gonçalves, J. L. M.; Raij, B.; Gonçalves, J. C. Florestais. In: Raij, B.; Catarella, H.; Quaggio, J. A.; Furlani, A. M. C. (Eds.). Recomendações de adubação e calagem para o Estado de São Paulo. 2. ed. Campinas: Instituto Agronômico de Campinas, 1996, p. 245-259.

Gustavsson, L.; Haus, S.; Ortiz, C. A.; Sathre, R.; Truong, N. L. Climate effects of bioenergy from forest residues in comparison to fossil energy. Applied Energy, v. 138, p. 36-50, 2015.

Hättenschwiler, S.; Jorgensen, H. B. Carbon quality rather than stoichiometry controls litter decomposition in a tropical rain forest. Journal of Ecology, v. 98, p. 754-763, 2010.

Hättenschwiler, S.; Coq, S.; Barantal, S.; Handa, I. T. Leaf traits and decomposition in tropical rainforests: Revisiting some commonly held views and towards a new hypothesis. New Phytologist, v. 189, p. 950965, 2011.

Hedwall, P. O.; Bergh, J.; Nordin, A. Nitrogenretention capacity in a fertilized forest after clear-cutting: The effect of forest-floor vegetation. Canadian Journal of Forest Research, v. 45, n. 1, p. 130-134, 2014. https://doi.org/10.1139/cjfr-2014-0281

Hérnandez, J.; Pino, A.; Salvo, L.; Arrarte, G. Nutrient export and harvst residue decomposition patterns of a Eucalyptus dunnii Maiden plantation in temperate climate of Uruguay. Forest Ecology and Management, v. 258, p. 92-99, 2009.

IEA - International Energy Agency. World energy outlook. Paris: IEA, 2011.

Kumaraswamy, S.; Mendham, D. S.; Grove, T. S.; O‘Connel, A. M.; Sankaran, K. V.; Rance, S. J. Harvest residue effects on soil organix matter, nutrientes and microbial biomass in eucalypt plantations in Kerala, India. Forest Ecology and Management, v. 328, p. 140149, 2014.

Laclau, J. P.; Ranger, J.; Deleporte, P.; Nouvellon, Y.; Saint-André, L.; Marlet, S.; Bouillet, J. P. Nutrient cycling in a clonal stand of Eucalyptus and an adjacent savanna ecosystem in Congo. 3. Input-output budgets and consequences for the sustainability of the plantations. Forest Ecology and Management, v. 210, p. 375-391, 2005.

Laclau, J. P.; Ranger, J.; Gonçalves, J. L. M.; Maquère, V.; Krusche, A. V.; M'Bou, A. T.; Nouvellon, Y.; Saint-André, L.; Bouillet, J. P.; Piccolo, M.C.; Deleporte, P. Biogeochemical cycles of nutrients in tropical Eucalyptus plantations. Main features shown by intensive monitoring in Congo and Brazil. Forest Ecology and Management, v. 259, p. 1771-1785, 2010.

Lal, R. Soil quality impact of residue removal for bioethanol production. Soil and Tillage Research, v. 102, n. 2, p. 233-241, 2009. https://doi.org/10.1016/j.still.2008.07.003

Mendes, J. C. T.; Seixas, F. Avaliação dos impactos da colheita florestal nos atributos físicos do solo em uma reserva legal. Scientia Forestalis, v. 46, n. 118, p. 143-155, 2018.

Mendham, D. S.; Sankaran, K. V.; O'Connell, A. M.; Grove, T. S. Eucalyptus globulos harvest residue management effects on soil carbon and microbial biomass at 1 and 5 years after plantations establishment. Soil Biology and Biochemistry, v. 34, p. 1903-1912, 2002. 
Mendham, D. S.; Kumaraswamy, S.; Sankaran, K. V.; John, K. S.; Grove, T. S.; O'Connell, A. M.; Rance, S. J.; Sujatha, M. P. An assessment of response of soil-based indicators to nitrogen fertilizer across four tropical Eucalyptus plantations. Journal of Forestry Research, v. 20 , n. 3, p. $237-242,2009$. https://doi.org/ 10.1007/s11676-009-0043-x

Mendham, D. S.; O'Connell, A. M.; Grove, T. S.; Rance, S. J. Residue management effects on soil carbon and nutrient contents and growth of second rotation eucalypts. Forest Ecology and Management, v. 181, n. 3, p. 357-372, 2003.

Mendham, D.; Orgden, G. N.; Short, T.; O'Connel, T. M.; Grove, T. S.; Rance, S. J. Repeated harvest residue removal reduces E. globulus productivity in the 3 rd rotation in South-Western Australia. Forest Ecology and Management, v. 329, p. 279-286, 2014.

Menegale, M. L. C.; Rocha, J. H. T.; Harrison, R.; Gonçalves, J. L. M.; Almeida, R. F.; Piccolo, M. C.; Hubner, A.; Junior, J. C. A.; Ferraz, A. V.; James, J. N.; Michelsen-Correa, S. Effect of timber harvest intensities and fertilizer application on stocks of soil C, N, P and S. $\begin{array}{llll}\text { Forests, } & \text { v. 7, } & \text { n. 12, 319, } 2016 .\end{array}$ https://doi.org/10.3390/f7120319

Mitchell, A. D.; Smethurst, P. J. Base cation availability and leaching after nitrogen fertilisation of a eucalypt plantation. Australian Journal of Soil Research, v. 46, n. 5 , p. $445-454$, 2008. https://doi.org/ 10.1071/SR08005

Moreira, F. M. S.; Siqueira, J. O. Microbiologia e bioquímica do solo. Lavras: UFLA, 2002.

Nambiar, E. K. S. Sustainability of eucalypt plantations in Australia is failing. Australian Forestry, v. 73, n. 4, p. 207-208, 2010. https://doi.org/10.1080/23256087.2010.12 002194

Nunez-Regueira, L.; Rodriguez-Anon, J. A.; Proupin, J.; Mourino, B.; Artiaga-Diaz, R. Energetic study of residual forest biomass using calorimetry and termal analysis. Journal of Thermal Analysis and Calorimetry, v. 80, p. 457-464, 2005. https://doi.org/10.1007/s10973-005-0677-7

Purahong, W.; Kapturska, D.; Pecyna, M. J.; Schulz, E.; Schloter, M.; Buscot, F.; Hofrichter, M.; Kreuger, D. Influence of different forest system management practices on leaf litter decomposition rates, nutrient dynamics and the activity of ligninolytic enzymes: a case study from central European forests. PLoS
ONE, v. 9, n. 4, e93700, 2014. https://doi.org/10.1371/journal.pone.0093700

Ranger, J.; Nys, C. Study of mineral elements in a coppice in the Ardennes (North of France): Balance for a complete rotation. Acta Oecologica, Oecologia Plantarum, v. 7, n. 3, p. 287-305, 1986.

Ring, E.; Jacobson, S.; Jansson, G.; Högbom, L. Effects of whole-tree harvst on soil-water chemistry at five conifer sites in Sweden. Canadian Journal of Forest Research, v. 47, p. 349-356, 2016.

Rocha, J. H. T; Marques, E. R. G.; Gonçalves, J. L. M; Hübner, A.; Brandani, C. B.; Ferraz, A. D. V.; Moreira, R. M. Decomposition rates of forest residues and soil fertility after clearcutting of Eucalyptus grandis stands in response to site and management and fertilizer application. Soil Use and Management, v. 32, p. 289-302, 2016.

Santana, R. C.; Barros, N. F.; Novais, R. F.; Leite, H. G.; Comerford, N. B. Alocação de nutrientes em plantios de eucalipto no Brasil. Revista Brasileira de Ciência do Solo, v. 32, n. spe., p. 2723-2733, 2008. https://doi.org/ 10.1590/S0100-06832008000700016

Sankaran, K. V.; Grove, T. S.; Kumaraswamy, S.; Manju, V. S.; Mendham, D. S.; O'Connell, A. M. Export of biomass and nutrients following harvest of eucalypt plantations in Kerala, India. Journal of Sustainable Forestry, v. 20 , no. 3, p. 15-36, 2005. https://doi.org/ 10.1300/J091v20n03_02

Schumacher, M. V.; Viera, M. Ciclagem de nutrientes em plantações de eucaliptos. In: Schumacher, M. V.; Viera, M. Silvicultura do eucalipto no Brasil. Santa Maria: Editora UFSM, 2015, p. 273-307.

Schumacher, M. V.; Caldeira, M. V. W. Biomass estimation and nutrient content of a Eucalyptus globulus (Labillardière) subespécie maidenii plantation. Ciência Florestal, v. 11, p. 45-53, 2001.

Silva, P. H. M.; Poggiani, F.; Libardi, P. L.; Gonçalves, A. N. Fertilizer management of eucalypt plantations on sandy soil in Brazil: Initial growth and nutrient cycling. Forest Ecology and Management, v. 301, p. 67-78, 2013.

Seixas, F. Efeitos físicos da colheita mecanizada de madeira sobre o solo. In: Gonçalves, J. L. M.; Stape, J. L. (Ed.). Conservação e cultivo de solos para plantações florestais. Piracicaba: IPEF, 2002. p. 131-204. 
Seixas, F.; McDonald, T. P.; Stokes, B. J.; Raper, R. L. Effect of slash on forwarder soil compaction. Procedings of XVIII Annual Meeting of the Council on Forest Engineering, Cashiers, p .77-86, 1995.

Thiffault, E.; Hannam, K. D.; Pare, D.; Titus, B. D.; Hazlett, P. W.; Maynard, D. G.; Brais, S. Effects of forest biomass harvesting on soil productivity in boreal and temperate forests: A review. Environmental Reviews, v. 19, p. 278-309, 2011. https://doi.org/10.1139/ A11-009

Vergutz, L.; Manzoni, S.; Porporato, A.; Novais, R .F.; Jackson, R. B. Global resorption efficiencies and concentrations of carbon and nutrients in leaves of terrestrial plants. Ecological Monographs, v. 82, p. 205-220, 2012. https://doi.org/10.1890/11-0416.1

Vos, M.; Lobo, A. B.; Jennings, S. J.; Kowalchuk, G. A. Micro-scale determinants of bacterial diversity in soil. FEMS Microbiology Reviews, v. 37, p. 936-954, 2013.

Witschoreck, R.; Schumacher, M. V. Alocação de nutrientes em povoamentos de Eucalyptus saligna Smith na região de Guaíba - Rio Grande do Sul. Cerne, v. 21, n. 4, p. 625-632, 2015.

Witschoreck, R. Recomendação de fertilizantes para Eucalyptus saligna Sm. com base no balanço nutricional na região de Guaíba-RS. Santa Maria: Universidade Federal de Santa Maria, 2014. (Tese de Doutorado).
Wronski, E. B. Logging trials near Tumut [New South Wales; trafficability of logging machines]. Australian Forest Industries Journal and Logger, v, 56, n. 4, p. 10-14, 1990.

Zeng, N.; King, A. W.; Zaitchik, B.; Wullschleger, S. D.; Gregg, J.; Wang, S.; KirkDavidoff, D. Carbon sequestration via wood harvest and storage: An assessment of its havest potential. Climate Change, v. 118, n. 2 , p. $245-257,2012$. https://doi.org/ 10.1007/s10584-012-0624-0

Zerpa, J. L.; Allen, H. L.; Campbell, R. G.; Phelan, J.; Duzan, H. Influence of variable organic matter retention on nutrient availability in a 10-year-old loblolly pine plantation. Forest Ecology and Management, v. 259, p. 1480-1489, 2010. 